\title{
Auxiliary Reflectance Sensor for Guided Surgery with Dental Implants: In Vitro Study
}

\author{
Diego Antonio Sigcho López ${ }^{1}$ Karina Pintaudi Amorim² Dalva Cruz Laganá2
}

${ }^{1}$ Faculty of Dentistry, Central University of Ecuador, Quito, Ecuador

${ }^{2}$ Faculty of Dentistry, University of São Paulo, São Paulo, Brazil
Address for correspondence Diego Antonio Sigcho López. PhD, Department of Prosthetic, Central University of Ecuador, Av. El Comercio E7-49 y el Tiempo, 170506, Quito, Ecuador (e-mail: diegosigcho@usp.br).

\author{
Abstract \\ Keywords \\ - computer-assisted \\ surgery \\ - dental implants \\ - ambulatory surgical \\ procedures \\ - tomography \\ - X-ray computed
}

Objectives The aim of this research was to develop a sensor of approximation by reflectance for guided surgery with dental implants without flap detachment, and verify the effectiveness of this system.

Materials and Methods Ten models of total edentulous arches were divided into two groups. Two implants of $3.5 \times 11.5 \mathrm{~mm}$ (NeoDent) were inserted in each model; in Group 1 (G1), a stereolithographic guide NeoGuide system was used. In Group 2 (G2), the experimental approximation sensor was used for the insertion of the implants. The evaluation of the results was performed by overlapping the virtual planning images with the tomographies of the models of the implants inserted.

Results There were no statistically significant differences between the guide and the sensor groups. The averages and standard deviations observed at the angulation of the guide was 4.15 ( 2.65 degrees) and 5.48 (2.85 degrees) at the sensor. The linear deviations at the cervical level were 0.002 (1.37) and $0.11(1.47) \mathrm{mm}$ and at the apical level $0.19(1.28)$ and $0.21(1.42) \mathrm{mm}$, respectively.

Conclusions The use of a guide is important for the stabilization of the drills; the greatest challenge is to control the apical position of the implants, especially in highly reabsorbed edges. The experimental sensor can become an auxiliary tool to the stereolithographic guides; however, several difficulties must still be overcome to recommend the use of a sensor.

\section{Introduction}

Computed tomography (CT) allows detailed plans of the jaws in all positions of the space; this orientation allows planning the insertion of dental implants with anatomical and prosthetic considerations. ${ }^{1}$ Nowadays, computer-aided design (CAD) and computer-aided manufacturing (CAM) in implantology allows the design of surgical guides to perform virtual planning in the jaws with greater accuracy.

There are two types of surgical guides, static and dynamic. Both use the CT data to plan the positioning of the implants. The static guides do not allow modification of the planning during their use; they are made with three-dimensional (3D) prototyping systems such as stereolithography. Dynamic guides are 3D navigation systems that allow the transfer of virtual planning directly to the jaws through a mechanical system or optical sensors that monitor the position of the surgical instrument in a real-time window. ${ }^{2}$

Guided surgery enables flapless surgery, which consists of a small incision on the bony crest, without detachment of the total mucoperiosteal flap, enough for the penetration of the drill for the perforation of the alveolar bone. ${ }^{3}$ This approach with limited gum removal can be applied with a circular scalpel at the drill insertion area or directly with the drill, initiating bone drilling. ${ }^{4}$ This technique decreases pain, bone loss, inflammatory process related to mucoperiosteal detachment, and improves postoperative phase and patient comfort. ${ }^{5}$ It avoids suture use, requires shorter surgical time, shorter healing time, enhances recovery, and produces less bleeding, thus facilitating immediate loading. ${ }^{6}$ 
Guided surgery can be limited in some cases; patients must have enough bone volume to be candidates for this technique. In cases where bone expansion is needed a sensor can be an auxiliary tool while drilling the bone, preventing fenestration of dental implants. ${ }^{6}$

\section{Materials and Methods}

Two systems were compared, a stereolithographic surgical guide (NeoDent; Industry and Trade of Dental Materials S.A. SP, Brazil), without the use of stabilizers from the Company kit, and an experimental reflectance sensor.

Ten models of acrylic resin with bone meal, bone and gum tissue simulators, prototypes, ${ }^{7}$ and identical copies of a single model were divided into two groups (-Fig. 1).

In Group 1 (G1), two implants were inserted in the anterior area in each model with the NeoGuide stereolithographic surgical guide, representing the control group. In Group 2 (G2), two implants were inserted with the assistance of

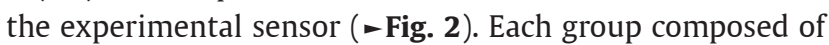
5 models with a total of 10 implants of $3.5 \times 11.5 \mathrm{~mm}$ Morse type, 2 in each model in the anterior area. The surgical area was determined by CT design of one of the casts. A single planning was performed for the entire sample (-Fig. $\mathbf{3}$ ).

The accuracy of the implants was tested using CT scans of each of the models after implant placement, superimposed on the initial planning. Deviations at cervical, apical, and angular orientation were evaluated.

\section{Composition of the Bone Material}

The models were composed of self-polymerized, chemically activated acrylic resin (Jet-Acrylic autopolymerizing) and bone meal (powdered fertilizer). The mixing ratio of the two materials was one part of acrylic resin ( $25 \mathrm{~g}$ of polymer) to three parts of bone meal $(75 \mathrm{~g}){ }^{7}$

The model was obtained from a total edentulous manikin that was worn out on the entire surface of the alveolar ridge and palate, which leaves the space for the layer of artificial gingiva, made by means of several perforations in the model of gypsum, with spherical drill, $2 \mathrm{~mm}$ in diameter. With a round cutter of tungsten carbide, several points were united in such a way that, in the end, the whole model had a wear of $2 \mathrm{~mm}$, area related to the gingival mucosa.

To simulate the mucosa on the models, they received a layer of soft comfort resin pressed inside a muffle, thus obtaining the experimental model (-Fig. 1).

\section{Tomography of the Experimental Model}

A randomized model was tomographed following the protocol of tomographic acquisition with Cone Bean Carestream CS9300, conducted at the Foundation of the Faculty of Dentistry of the University of São Paulo (FUNDECTO), with a field of view (FOV) of $5 \times 5,75 \mathrm{Kv}, 4.0 \mathrm{~mA}$ in 20 seconds and a voxel of $90 \mu \mathrm{m}$. These data were sent to the NeoDent Company for the preparation of NeoGuide, with the planning defined for placement of two implants in the canine regions (13 and 23; -Fig. 3).

\section{Experimental Sensor by Digital Reflectance}

The Pololu QTR-8A [http://www.pololu.com/product/960] and QTR-8RC [http://www.pololu.com/product/961] reflectance sensor sets, proximity reflection of general use, were tested. Each module is a convenient support for eight pairs of infrared (IR) emitters and phototransistors uniformly
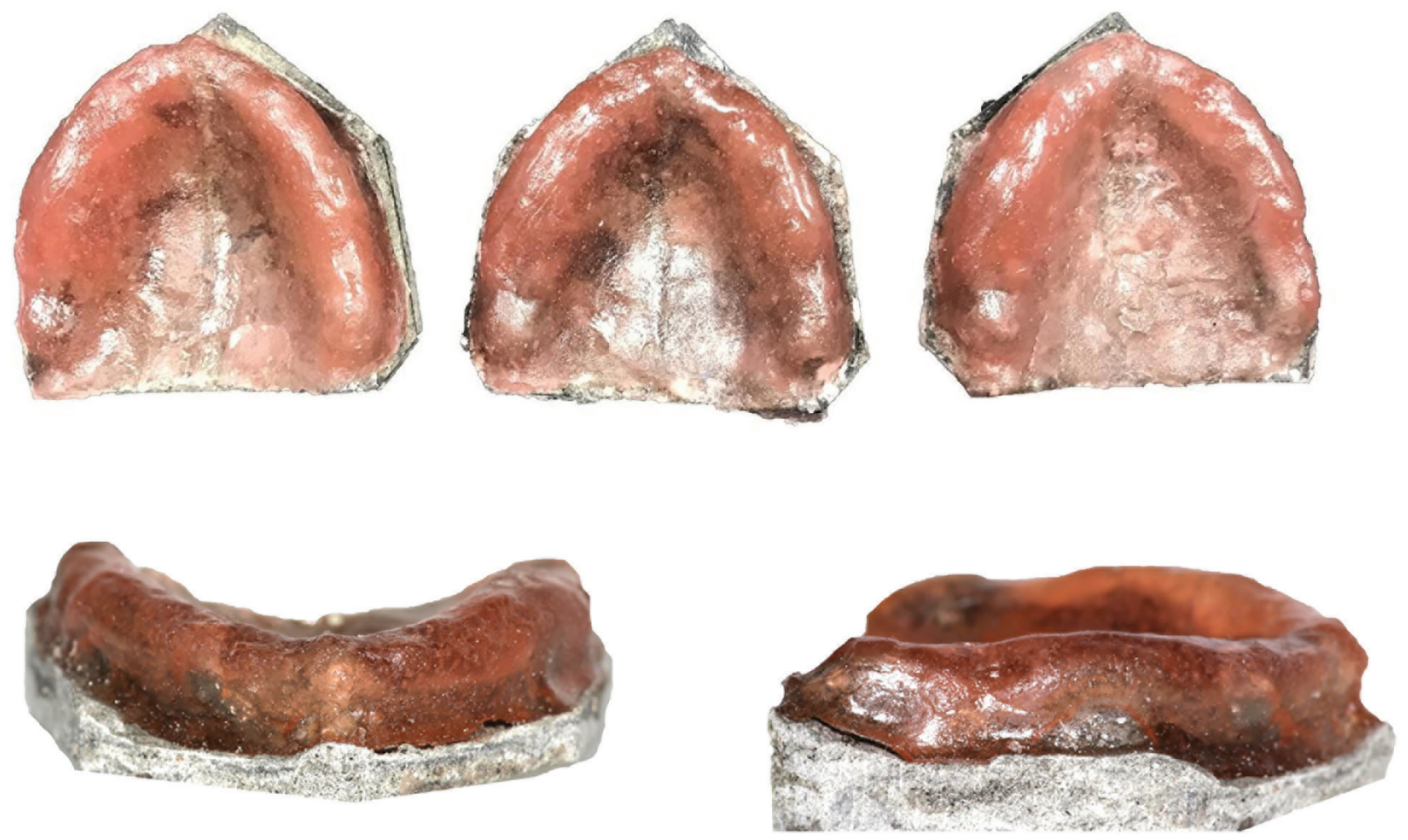

Fig. 1 Maxillary simulators, prototypes patented by Novellino and Laganá. ${ }^{7}$ 


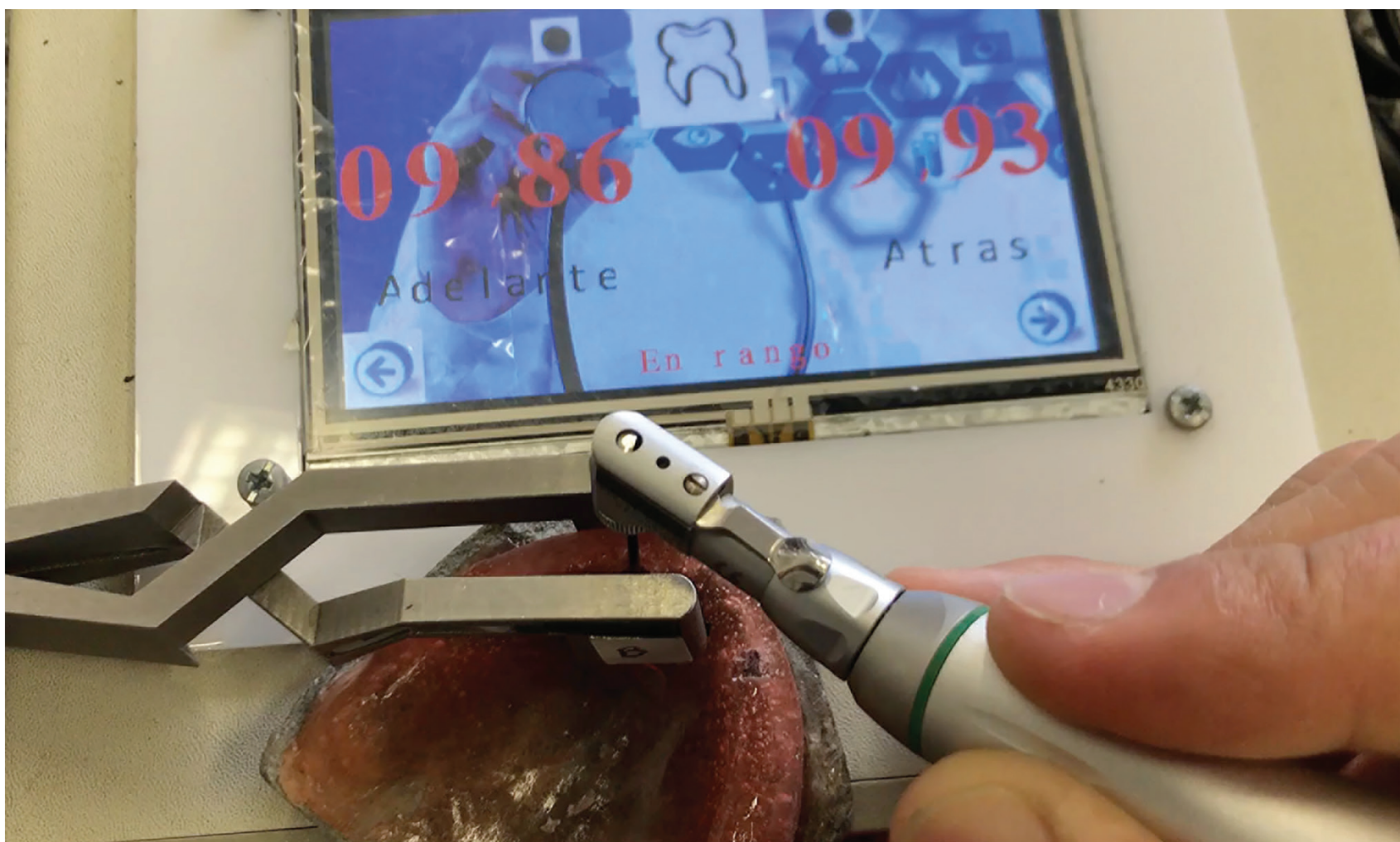

Fig. 2 Experimental reflectance approximation sensor.

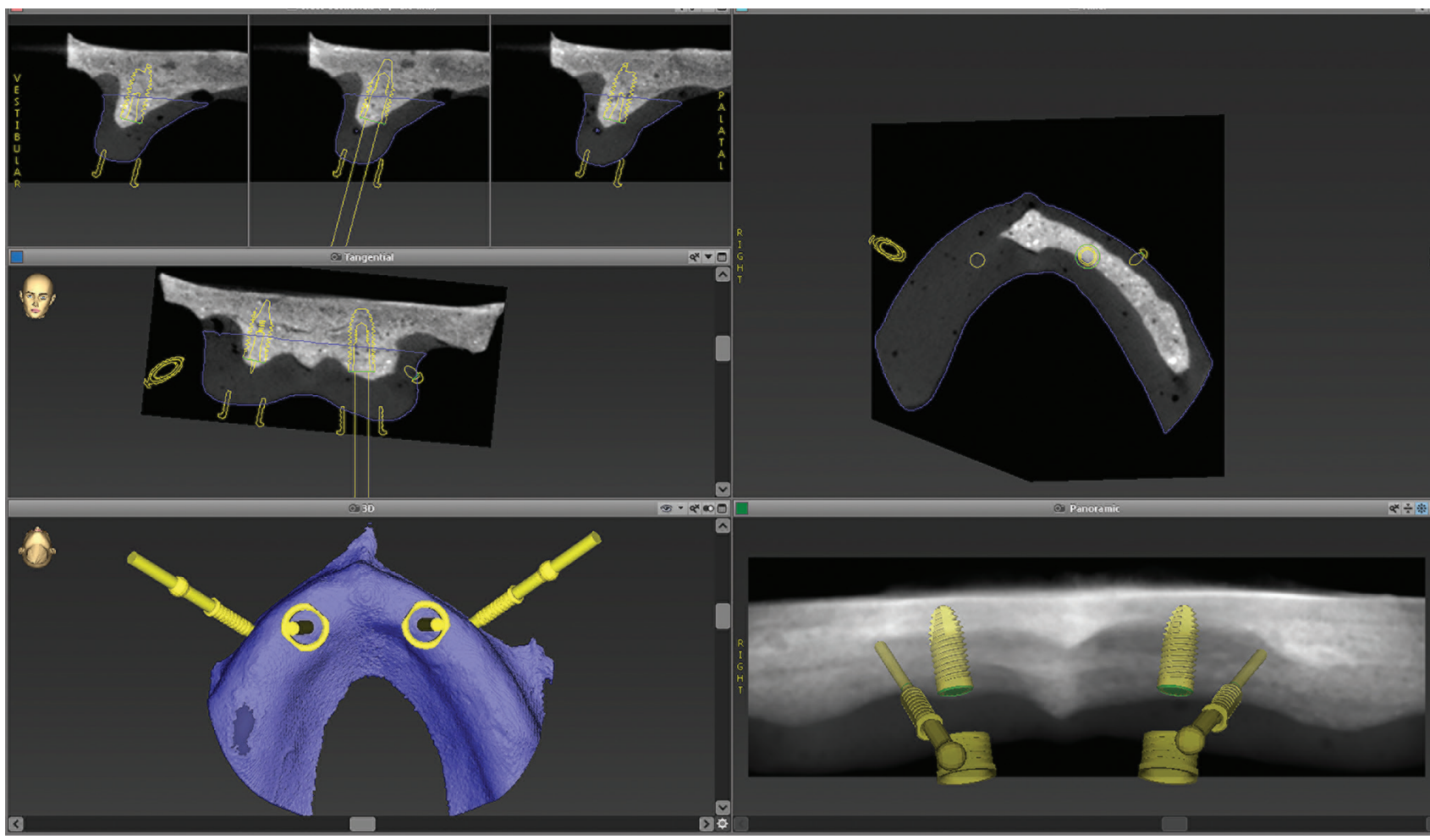

Fig. 3 Digital planning by the NeoDent team.

spaced apart at intervals. The outputs are independent, but the light-emitting diodes (LEDs) are arranged in pairs to reduce power consumption by half. LEDs are controlled by a metal-oxide-semiconductor-field-effect transistor (MOSFET; TECMOS) with a normally pulled high gate, allowing the
LEDs to be turned off by configuring the MOSFET gate for low voltage.

LED current limiting resistors for $5 \mathrm{~V}$ operation are arranged in two stages. This allows a simple one-stage bypass to enable $3.3 \mathrm{~V}$ operation. The LED's current is 
approximately 20 to $25 \mathrm{~mA}$, making the total board consumption a little below $100 \mathrm{Ma}$. A switch to turn off and on the sensors A and B was added to a clamping clamp (-Fig. 2).

\section{Insertion of Implants}

In Group 1, according to the protocol, two fixation pins were used to keep the surgical guide in place. Subsequently, the 2.0 and 3.5 sleeves of the NeoGuide kit for the drilling bits were inserted into the guide (-Figs. 4 and 5 ).

In Group 2, a circular incision was made at the cervical level with assistance of NeoGuide (-Fig. 6). The drill sequence was the same as in G1, but with drill bits from the surgical kit and the sensor instead of the stereolithographic guide (-Fig. 2). The implants were inserted by free hand without the guide or sensor.

The accuracy of the implants was tested by tomography of each model after implants' placement, superimposing the initial virtual planning. Deviations at the cervical, apical, and angular levels were evaluated by co-diagnostic $\mathrm{X}$ software.

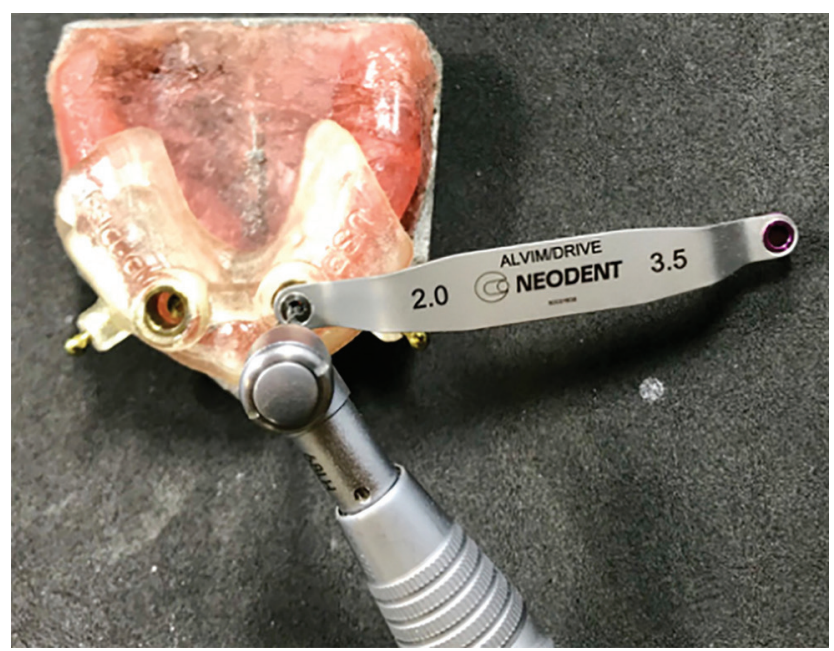

Fig. 4 Fixing pins positioned on the buccal wall and guide to the drill 2.0 NeoGuide.

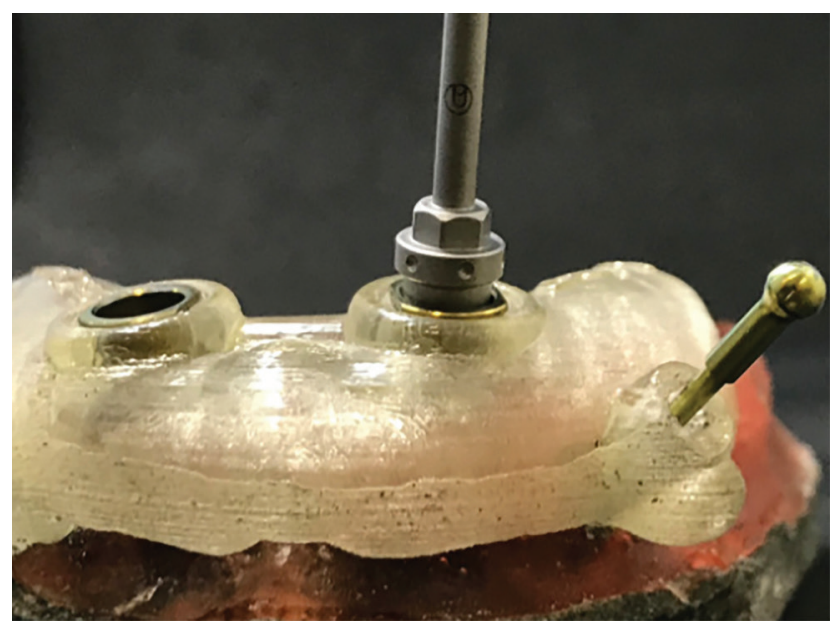

Fig. 5 Insertion of implant $3.5 \mathrm{~mm}$ diameter $\times 11.5 \mathrm{~mm}$ long drive cone Morse with $50 \mathrm{~N}$ with the guide NeoGuide (G1).

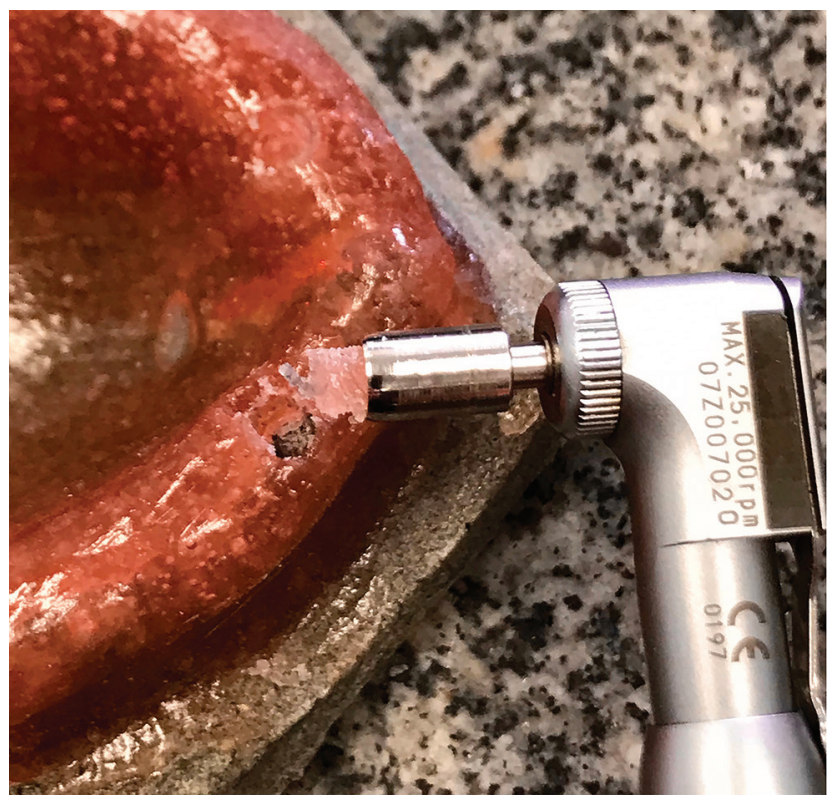

Fig. 6 Circular incision at cervical level, marking the beginning of G2 perforation.

\section{Results}

The evaluations of tomographic images on the software are shown in - Table 1.

The Shapiro Wilk statistical test was used to evaluate the normality of the data obtained, with the sample tending to normality.

The statistical analysis of the data was performed in two steps: one for linear deviations between the planned and executed, and another to analyze the angular discrepancies. Student's $t$-test was performed for parametric independent groups (-Table 2 ).

The results of the analysis of variance for the linear deviations and angulations showed no statistically significant difference between the groups.

The consistency and hardness of the models were higher than those found in the natural bone type IV. Therefore, greater force was used during the perforation and implant insertion, which may influence the micromovement of the surgical guide.

The experimental digital reflectance sensor presented some difficulties and technical errors, among them a delay in displaying the measurement; the operator needed to alternate his attention between the sensor screen and the drills in relation to the sensor tips.

The choice of the clamp used to fix the sensor was not adequate due to its lack of anatomy, making it difficult to position the sensor.

In - Fig. 7, a greater deviation can be observed in G2, with the sensor; however, the statistical differences were not significant between the groups.

- Figure 8 shows a slightly greater range of deviations in $\mathrm{G} 2$, with the sensor, but the differences were not significant between the groups.

- Figure 9 shows a slightly lower range of deviations in G2, with the sensor, but the differences were not significant 
Table 1 Angular and linear deviations obtained by the overlapping of the tomographic images of the installed implants in relation to the virtual planning

\begin{tabular}{|c|c|c|c|c|c|c|c|}
\hline Sagittal plane & & & & $\begin{array}{l}\text { Coronal } \\
\text { plane }\end{array}$ & & & \\
\hline Implant N 13 & $\begin{array}{l}\text { Angle } \\
\text { deviation } \\
\text { (degree) }\end{array}$ & $\begin{array}{l}\text { Entry } \\
(\mathrm{mm})\end{array}$ & $\begin{array}{l}\text { Apical } \\
(\mathrm{mm})\end{array}$ & Implant N 13 & $\begin{array}{l}\text { Angle deviation } \\
\text { (degree) }\end{array}$ & Entry (mm) & $\begin{array}{l}\text { Apical } \\
(\mathrm{mm})\end{array}$ \\
\hline 1 & 6.6 & -1.13 & 0.2 & 1 & 6.6 & -1.2 & -1.2 \\
\hline 2 & 1.2 & 1.1 & 0.87 & 2 & 1.2 & -0.8 & -0.88 \\
\hline 3 & 2.8 & -0.77 & -0.3 & 3 & 2.8 & -0.27 & 0.02 \\
\hline 4 & 2.5 & -2.27 & -1.76 & 4 & 2.5 & 0.36 & 0.3 \\
\hline 5 & 4.5 & -2.66 & -2.01 & 5 & 4.5 & -0.47 & -1.1 \\
\hline 6 & 6.2 & -2.19 & -2.87 & 6 & 6.2 & -1.06 & -0.02 \\
\hline 7 & 3 & 0.73 & 0.24 & 7 & 3 & 0.04 & 0.37 \\
\hline 8 & 6.6 & -0.93 & 0.28 & 8 & 6.6 & -1.73 & -1.22 \\
\hline 9 & 10.9 & -1.65 & 0.48 & 9 & 10.9 & 0.86 & 0.37 \\
\hline 10 & 7.5 & -1.24 & 0.08 & 10 & 7.5 & -0.23 & 0.47 \\
\hline Sagittal plane & & & & Coronal plane & & & \\
\hline Implant N 23 & $\begin{array}{l}\text { Angle } \\
\text { deviation } \\
\text { (degree) }\end{array}$ & $\begin{array}{l}\text { Entry } \\
(\mathrm{mm})\end{array}$ & Apical (mm) & Implant N 23 & $\begin{array}{l}\text { Angle deviation } \\
\text { (degree) }\end{array}$ & Entry (mm) & Apical (mm) \\
\hline 1 & 9.5 & 2.3 & 0.41 & 1 & 9.5 & 0.76 & 0.89 \\
\hline 2 & 1.6 & 1.45 & 1.28 & 2 & 1.6 & 0.38 & 0.65 \\
\hline 3 & 6 & 2.56 & 3.44 & 3 & 6 & 0.12 & 0.95 \\
\hline 4 & 1.4 & -1.12 & -1.06 & 4 & 1.4 & 1.31 & 1.59 \\
\hline 5 & 5.4 & -0.19 & 0.87 & 5 & 5.4 & 0.58 & 0.82 \\
\hline 6 & 4.4 & 3.27 & 4.01 & 6 & 4.4 & 1.33 & 1.81 \\
\hline 7 & 8.1 & 1.94 & 0.4 & 7 & 8.1 & 0.18 & -0.31 \\
\hline 8 & 2.8 & -0.69 & -0.18 & 8 & 2.8 & 0.22 & 0.44 \\
\hline 9 & 4.2 & -0.99 & -0.16 & 9 & 4.2 & 0.73 & 0.56 \\
\hline 10 & 1.1 & 2.55 & 2.5 & 10 & 1.1 & 1.22 & 0.99 \\
\hline \multicolumn{8}{|c|}{ Guide (1-5) } \\
\hline \multicolumn{8}{|c|}{ Sensor (6-10) } \\
\hline
\end{tabular}

between the groups. Extreme atypical values were observed in the G2 (sensor) represented by the points at the ends of the sensor results box.

\section{Discussion}

Implant survival may be affected by flap detachment as bone resorption is increased. Thus, when guided surgery is not possible, a tool calibrated and based on CT could meet the surgical needs. ${ }^{8}$

When comparing the results obtained in the reviews of Di Giacomo et $\mathrm{al}^{9}$ and further work by Vercruyssen et $\mathrm{al}^{2}$ with smaller deviations, the progress of the technique can be observed. Even so, the current deviations suggest the need for research to obtain an increasingly accurate system. ${ }^{10}$

Our results support the findings of other studies with linear and angular deviations of less than $1 \mathrm{~mm}$ and angle of 4 degrees (-Table 2). Among stereolithographic guides, Zhou et $\mathrm{al}^{10}$ observed $1.25 \mathrm{~mm}$ (95\% confidence interval [CI]: $1.22-$ 1.29) at the cervical level, $1.57 \mathrm{~mm}$ (95\% CI: 1.53-1.62) at apex, and 4.1 degrees (95\% CI: 3.97-4.23) at the angular slope. They also observed a significant association between bone density and deviation, with results in greater precision in the mandible when compared with the maxilla and when using fixation. In this study, we expect higher deviations because of model's density, which was greater than human bone; therefore, extra force was needed and micro movements were possible.

By comparing the variations between the guided system (G1) and the sensor (G2), it could be observed that the implants placed with the sensor showed greater intervals (-Figs. 7 and 8 ) except for entry to the cervical level (-Fig. 9), since the beginning of the drilling was marked with the stereolithographic guide at the entry point. However, the sensor at the cervical level presented extreme values, possibly due to sliding of the drills or changes in the shape of the artificial gingiva. The sensor could be associated with 
Table 2 Summary of descriptive statistical analysis and Student's $t$-test

\begin{tabular}{|c|c|c|c|c|}
\hline Deviations & Guide & Sensor & Student's $t$-test $U$ & Student's $t$-test B \\
\hline Angulação & & & $p$-Value (unilateral) = & $p$-Value $($ bilateral) $=$ \\
\hline Mean & 4.15 & 5.48 & 0.0677 & 0.1354 \\
\hline SD & 2.6566 & 2.8547 & & \\
\hline Max. & 9.5 & 10.9 & & \\
\hline Min. & 1.2 & 1.1 & & \\
\hline Entry & & & p-Value (unilateral) = & $\mathrm{p}$-Value (bilateral) = \\
\hline Mean & 0.002 & 0.118 & 0.3992 & 0.7983 \\
\hline SD & 1.373 & 1.476 & & \\
\hline Max. & 2.56 & 3.27 & & \\
\hline Min. & -2.66 & -2.19 & & \\
\hline Apical & & & p-Value (unilateral) = & $\mathrm{p}$-Value (bilateral) $=$ \\
\hline Mean & 0.199 & 0.213 & 0.4871 & 0.9742 \\
\hline SD & 1.289 & 1.429 & & \\
\hline Max. & 3.44 & 3.27 & & \\
\hline Min. & -2.01 & -2.19 & & \\
\hline
\end{tabular}

Abbreviations: Max., maximum; Min., minimum; SD, standard deviation.

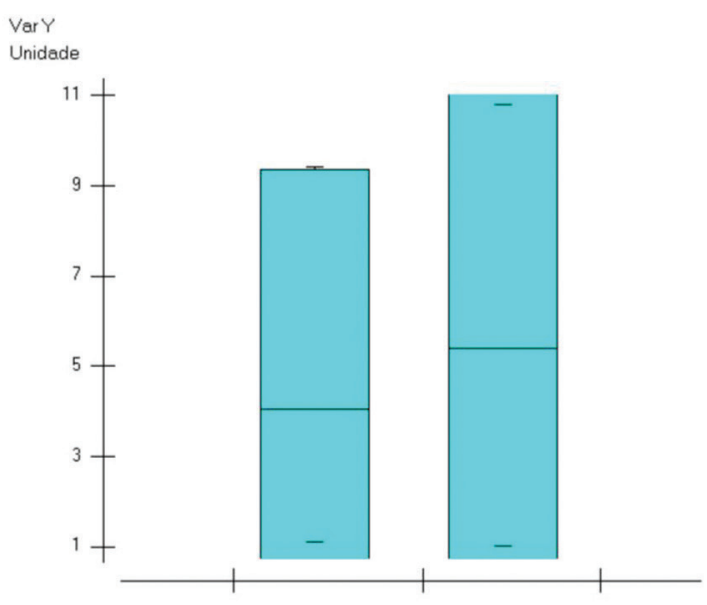

Fig. 7 Box plot of statistical analysis of Student's t-test showing averages of angular slopes between G1 and G2.

a stereolithographic guide to limit the deviations, not being safe to operate this device by itself.

The reflectance sensor is a dynamic guide and the observed results are also close to those mentioned in the review by Block and Emery ${ }^{11}$ with guides approximately $0.4 \mathrm{~mm}$ at the entrance and 4 degrees of angulation (-Table 2 ).

The simulated bone density obtained in this study was similar to dense type I bone; the acrylic matrix caused higher density than expected, with 2,200 HU radiographically, but the manual sensed density during drilling was greater. In G2, where it was not NeoGuide guide, there was a slipping with the use of the spear-type drill due to the density of the

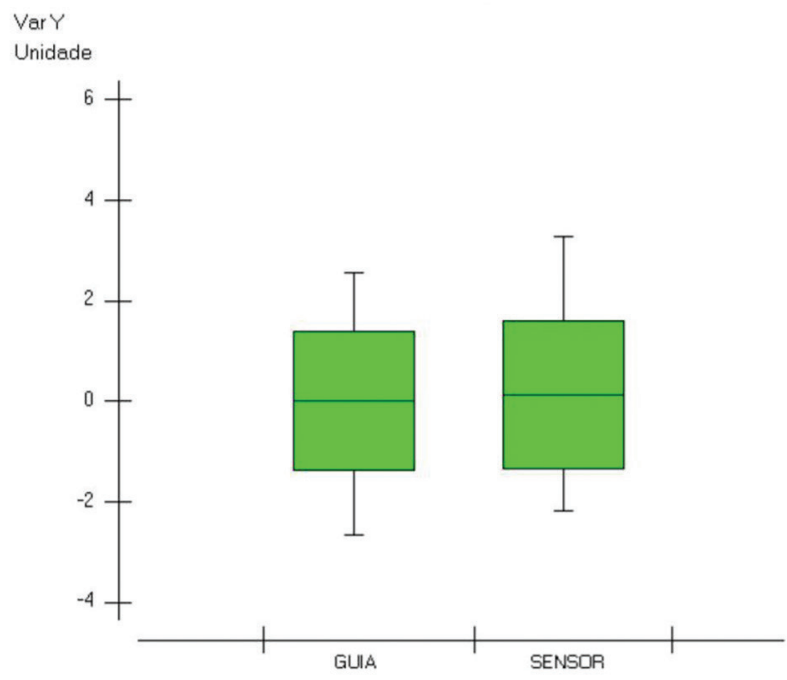

Fig. 8 Box plot of statistical analysis of Student's $t$-test showing averages at the cervical level, the entry of the drills between G1 and G2. A longer interval can be observed in G2.

experimental model during the first drilling, making it necessary to apply extra force to overcome the strength of the structure. Contrary to clinical findings, in which the maxilla was subject to greater deviations when compared with the mandible, ${ }^{10}$ bone density may be a factor associated with deviations, in type I and IV bones. Similar values were found in the systematic review of Seo and Juodzbalys ${ }^{12}$ with maximum deviation of $2.19 \mathrm{~mm}$ at the apical level, $1.68 \mathrm{~mm}$ at the coronal level, and angular inclination of 4.67 degrees in edentulous patients. 


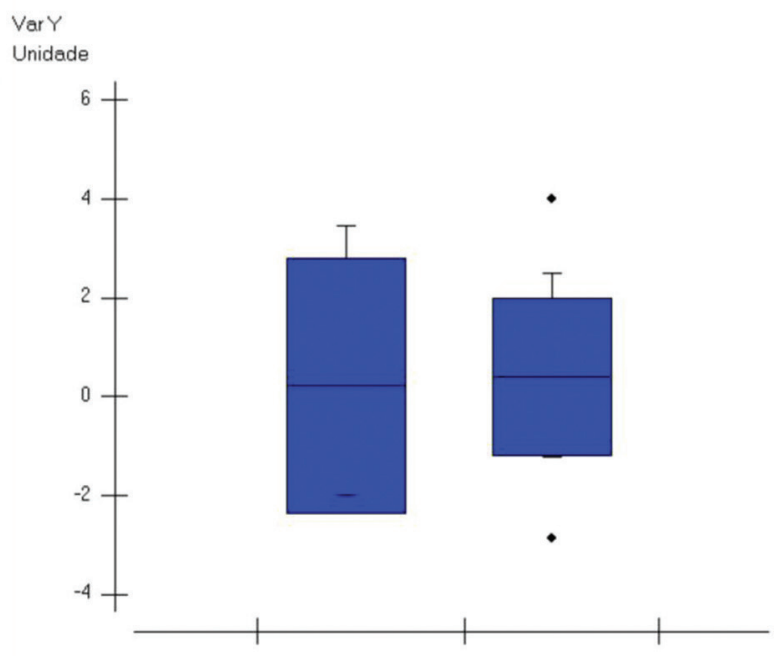

Fig. 9 Box plot of statistical analysis of Student's $t$-test showing averages at the apical level, the entry of the drills between G1 and G2.

In G1, stereolithographic guide kept the drills firmly, decreasing time and facilitating procedures considerably. Another advantage of the NeoGuide system is that it allows to see if there is a deviation during insertion of the implant when approaching the sleeves of the positioner and the guide ( - Fig. 5). By observing this relation, the position of the implant can be corrected by drilling.

It is important to consider that in G1, the stabilizers of NeoGuide system, a differential of the NeoDent Company, were not used, and that considerably increases the implants' accuracy. Results could not be compared with the majority of systems that do not have these parts. This explains the similar results between the G1 and the sensor by reflectance. However, the sensor can obtain results similar to a common prototyped guide, but would hardly achieve the results of NeoGuide or other commercial guides.

Few laboratory studies are reported in the literature regarding surgical and alternative techniques. The findings in this study corroborate with the findings of Kang et $\mathrm{al}^{13}$ preferring the stereolithographic guides over the dynamic systems. By limiting the displacement of the drills, the technique is safe and easy to perform.

During the drilling of the bone in G2, with the experimental sensor, difficulty in keeping the drills in a stable position was perceived. The initial drill slid and varied from the irregular topography of the experimental model. Similar difficulties are to be expected without the guided surgery kit system that keeps the drill firm and in position. The deviations found in this work are also related to the model used, which, in addition to being denser than the bone, had a fine alveolar ridge, an unfavorable characteristic for guided surgery. Among the difficulties observed regarding the use of the experimental sensor by digital reflectance, the alternation of attention between the insertion site and the sensor screen may produce drawbacks during drilling. This distraction was also observed in dynamic systems. ${ }^{14}$

Van de Velde et $\mathrm{al}^{15}$ reported frequent bone perforations in the surgeries without flap detachment and without the use of surgical guides. Stereolithographic guides without a metal ring and the system of components for guided surgery are at higher risk of significant deviations in the final position of the implants due to micro movements.

Vercruyssen et al ${ }^{16}$ suggested that the reduction of steps in the preoperative guided surgery could improve the accuracy of surgical guides, associated with optical scanners and 3D print enhancements. A sensor associated with the stereolithographic guide could reduce and even eliminate the risks and deviations reported in the literature. ${ }^{17}$

It is necessary to emphasize that this was the first experiment related to a digital reflectance sensor for implant installation. Several difficulties appeared during the use of the sensor, among them the delay in display the accurate distance of the drill bit, so the expected alarm to avoid perforations may be late. This delay also increased the drilling time.

Specifically, it is necessary to change the gripper since the one used in G2 was unstable. Although the experimental sensor did not present better results compared with the stereolithographic guide, it was an important step in the development of a new tool in the near future. Surgical guides facilitate the insertion of dental implants with greater safety for the surgeon and less trauma for the patient.

The major deviations in the guided surgery system appear at the apex of the implants,,$^{10}$ since the greatest control of the guides is at the cervical level at the entrance of the drills (-Table 2). The observed deviations are probably related to the size of the guide, which covered a small area of the jaw fixed with two lateral buccal pins.

In total edentulous jaws it was recommended that the guide covers most of the space and fixed with two anterior and two posterior pins. ${ }^{18,19}$

The development of a locator for the drills within the bone can be of great benefit, both with and without the detachment of the flap surgery.

Several difficulties were encountered during its use. It is necessary to create anatomical tweezers to adapt the sensors to the jaws and obtain greater precision. The major challenge is to control the apical position of the implants, especially in highly reabsorbed edges.

\section{Conclusions}

According to the proposal of this research, the experimental sensor by digital reflectance in the conditions of the current experiment is not recommended for clinical use; however, the sensor can be an auxiliary tool to the stereolithographic guides. We found stereolithographic surgery easier, faster, and safer than the experimental guide.

\section{Conflict of Interest}

None declared.

\section{Acknowledgments}

The authors would like to acknowledge the Faculty of Dentistry of University of Sao Paulo, Faculty of Dentistry of Central University of Ecuador, and NeoDent Company. The sensor was developed by Engineer Pablo Valdivieso in Ecuador. 


\section{References}

1 D'haese J, Van De Velde T, Elaut L, De Bruyn H. A prospective study on the accuracy of mucosally supported stereolithographic surgical guides in fully edentulous maxillae. Clin Implant Dent Relat Res 2012;14(2):293-303

2 Vercruyssen M, Hultin M, Van Assche N, Svensson K, Naert I, Quirynen M. Guided surgery: accuracy and efficacy. Periodontol 2000 2014;66(1):228-246

3 Brodala N. Flapless surgery and its effect on dental implant outcomes. Int J Oral Maxillofac Implants 2009;24 (Suppl) :118-125

4 Voulgarakis A, Strub JR, Att W. Outcomes of implants placed with three different flapless surgical procedures: a systematic review. Int J Oral Maxillofac Surg 2014;43(4):476-486

5 Arisan V, Karabuda ZC, Ozdemir T. Accuracy of two stereolithographic guide systems for computer-aided implant placement: a computed tomography-based clinical comparative study. J Periodontol 2010;81(1):43-51

6 Rocci A, Rocci M, Scoccia A, Martignoni M, Gottlow J, Sennerby L. Immediate loading of maxillary prostheses using flapless surgery, implant placement in predetermined positions, and prefabricated provisional restorations. Part 2: a retrospective 10-year clinical study. Int J Oral Maxillofac Implants 2012;27(5):1199-1204

7 Novellino MM, Sesma N, Laganá DC, Ferrari G. Linear and angular deviations of implants placed in experimental casts with stereolithographic drill guides fixed by o'ring ortho implant devices. Braz Dent J 2013;24(4):391-396

8 Pozzi A, Tallarico M, Marchetti M, Scarfò B, Esposito M. Computer-guided versus free-hand placement of immediately loaded dental implants: 1-year post-loading results of a multicentre randomised controlled trial. Eur J Oral Implantology 2014;7(3):229-242

9 Di Giacomo GA, Cury PR, de Araujo NS, Sendyk WR, Sendyk CL. Clinical application of stereolithographic surgical guides for implant placement: preliminary results. J Periodontol 2005;76(4):503-507
10 Zhou W, Liu Z, Song L, Kuo CL, Shafer DM. Clinical factors affecting the accuracy of guided implant surgery-a systematic review and meta-analysis. J Evid Based Dent Pract 2018;18(1):28-40

11 Block MS, Emery RW. Static or dynamic navigation for implant placement-choosing the method of guidance. J Oral Maxillofac Surg 2016;74(2):269-277

12 Seo C, Juodzbalys G. Accuracy of guided surgery via stereolithographic mucosa-supported surgical guide in implant surgery for edentulous patient: a systematic review. J Oral Maxillofac Res 2018;9(1):e1

13 Kang SH, Lee JW, Lim SH, Kim YH, Kim MK. Verification of the usability of a navigation method in dental implant surgery: in vitro comparison with the stereolithographic surgical guide template method. J Craniomaxillofac Surg 2014;42(7):1530-1535

14 Wanschitz F, Birkfellner W, Figl M, et al. Computer-enhanced stereoscopic vision in a head-mounted display for oral implant surgery. Clin Oral Implants Res 2002;13(6):610-616

15 Van de Velde T, Glor F, De Bruyn H. A model study on flapless implant placement by clinicians with a different experience level in implant surgery. Clin Oral Implants Res 2008;19(1):66-72

16 Vercruyssen M, Coucke W, Naert I, Jacobs R, Teughels W, Quirynen M. Depth and lateral deviations in guided implant surgery: an RCT comparing guided surgery with mental navigation or the use of a pilot-drill template. Clin Oral Implants Res 2015;26(11):1315-1320

17 Tatakis DN, Chien HH, Parashis AO. Guided implant surgery risks and their prevention. Periodontol 2000 2019;81(1):194-208

18 Marlière DAA, Demètrio MS, Picinini LS, Oliveira RG, Netto HDMC. Accuracy of computer-guided surgery for dental implant placement in fully edentulous patients: a systematic review. Eur J Dent 2018;12(1):153-160

19 D'haese J, Van De Velde T, Elaut L, De Bruyn H. A prospective study on the accuracy of mucosally supported stereolithographic surgical guides in fully edentulous maxillae. Clin Implant Dent Relat Res 2012;14(2):293-303 